\title{
Molecular signature of gastric cancer progression in clinical using whole genome sequencing and the Cancer Genome Atlas (TCGA) analysis
}

\author{
Gai-Gai Wei ${ }^{1,2 \#}$, Xiao-Chuan Geng ${ }^{3 \#}$, Ming-Sheng Fu ${ }^{4}$, Jun Wei ${ }^{5}$, Hai-Bo Yu ${ }^{6}$, Xiao-Hui Li $^{7}$, \\ Gang-Long Gao ${ }^{1,5,8}$
}

${ }^{1}$ Department of General Surgery, Renji Hospital, School of Medicine, Shanghai Jiao Tong University, Shanghai, China; ${ }^{2}$ Children's Hospital of Fudan University, National Children's Medical Center, Shanghai, China; ${ }^{3}$ Department of Radiology, Renji Hospital, School of Medicine, Shanghai Jiao Tong University, Shanghai, China; ${ }^{4}$ Department of Gastroenterology, Shanghai Fifth People’s Hospital, Fudan University, Shanghai, China; ${ }^{5}$ Department of General Surgery, Shanghai Fengxian District Central Hospital, Shanghai, China; ${ }^{6}$ Department of Hepatobiliary Surgery, Wenzhou Central Hospital, Wenzhou Medical University, Wenzhou, China; ${ }^{7}$ Department of Radiology, Children's Hospital of Nanjing Medical University, Nanjing, China; ${ }^{8}$ Department of Biliary and Pancreatic Surgery, Renji Hospital, School of Medicine, Shanghai Jiao Tong University, Shanghai, China Contributions: (I) Conception and design: GG Wei, GL Gao; (II) Administrative support: GG Wei, XC Geng, GL Gao; (III) Provision of study materials or patients: XC Geng, MS Fu, XH Li; (IV) Collection and assembly of data: J Wei, HB Yu; (V) Data analysis and interpretation: GG Wei, XC Geng, XH Li; (VI) Manuscript writing: All authors; (VII) Final approval of manuscript: All authors.

\#These authors contributed equally to this work.

Correspondence to: Gang-Long Gao. Department of General Surgery, Renji Hospital, School of Medicine, Shanghai Jiao Tong University, Shanghai, China. Email: ggl149@126.com; Xiao-Hui Li. Department of Radiology, Children's Hospital of Nanjing Medical University, Nanjing, China. Email: lixiaohui197846@sohu.com.

Background: The stomach adenocarcinoma (STAD) is one of the most common malignancies and the 5 -year survival rate of patients is very low. Therefore, clarify the whole mechanism of STAD in the molecular biology level is an urgent need to get a better prognostic outcome of STAD patients.

Methods: Two gastric cancer and two adjacent tissues from two STAD patients were subjected to whole genome sequencing (WGS). Based on the comparison of the Cancer Genome Atlas (TCGA) and our own WGS data of STAD, the survival prognosis and the shared single nucleotide variant (SNV) were analyzed.

Results: We identified a total of 1,191 differentially expressed genes (DEGs) between gastric cancer and adjacent tissues which are mainly enriched in the DNA replication, cell adhesion, digestive tract development, and serine-type peptidase activity pathway or related functions. Moreover, high levels of proline rich 15 like (PRR15L), human riboflavin transporter-3 (SLC52A3) and spindle and kinetochore associated complex subunit 1 (SKA1) were positively correlated with the high survival rate. The amplifications in 9p, 17q, 19q, and deletions in 4q, 6p, and 18q were more significant than other chromosomes. In addition, TTN, MUC16, and FLG showed the expression changed in most samples in TCGA STAD data and our own samples.

Conclusions: PRR15L and SLC52A3 were the potential prognostic biomarkers for STAD. TTN, MUC16, and FLG were upregulated and related to cancer development in STAD, these genes may serve as potential targets for gene therapy in STAD.

Keywords: Gastric cancer; cell adhesion; proline rich 15 like (PRR15L); human riboflavin transporter-3 (SLC52A3); spindle and kinetochore associated complex subunit 1 (SKA1)

Submitted Jun 11, 2021. Accepted for publication Sep 03, 2021.

doi: $10.21037 /$ tcr-21-1011

View this article at: https://dx.doi.org/10.21037/tcr-21-1011 


\section{Introduction}

The stomach adenocarcinoma (STAD) is one of the most common malignancies (1), and the 5 -year survival rate of patients with advanced STAD is very low (2). At present, the mechanism involved in tumorigenesis of gastric cancer remains unclear, and there is no effective marker to predict the occurrence and progression of gastric cancer (3). Therefore, an in-depth study to figure out the whole picture of STAD at the molecular level is an urgent need to get a better prognostic outcome of STAD patients.

In this study, we try to figure out potential gene therapy targets in STAD. First, the Cancer Genome Atlas (TCGA) database provides the information of the copy number variations (CNVs), the mRNA expression, single nucleotide variant (SNV), and the molecular signaling pathways which relate to candidate genes in various tumors and the para-cancer tissues $(4,5)$. TCGA database is not only an authoritative resource for basic, translational, and clinical studies but also have the potential to develop new target for cancer diagnostic and therapeutic strategies (6). $\mathrm{CNV}$ also known as copy number polymorphisms (CNPs), refers to genome structural variations, contained euploid or aneuploidy variations, amplification, and deletion. CNVs of several important molecules have been shown to play the critical role in cancer diagnosis, prognosis and therapy (7). For example, Hackmann et al. reported that copy number detection is a crucial analysis in hereditary breast and ovarian cancer (7). Besides, it has also been reported that widespread somatic Long INterspersed Element-1 (L1) insertions occur in the early stage of gastrointestinal cancer (8).

Plenty of studies have demonstrated that SNV is closely related to cancer progression. Kambayashi et al. reported that a rare variant of TP53 single nucleotide polymorphism was associated with the advanced invasive extramammary Paget's disease concomitant with cecal cancer possessing (9). To investigate the molecular role of genes in STAD, a study based on the 153 gastric cancer patients assessed SNVs, InDels (insertion or deletion of bases in the genome) and tumor mutation burden (TMB) of tumors by BTC-PCPD panel, and demonstrated the utility of massively parallel DNA sequencing of tumors to guide clinical management (10). In addition, an SNV in microRNA-1269a promotes the occurrence and process of hepatocellular carcinoma by targeting oncogenes SPATS2L and LRP6 (11). These studies suggested that the identification of SNV and CNVs of tumor-related genes is critical for their applications in cancer therapy in the future. In our study, based on the data from TCGA and our own sequencing data of STAD, the mRNA expression, CNVs, and SNV were systematically analyzed and try to figure out gene therapy targets from these results.

In this study, we hypothesized that if the same mutations occur in most samples in TCGA, and such mutations are associated with gastric cancer. Finally, based on the number of mutant samples, the heatmap was carried out for the top 20 mutant genes. the SNV results both in the TCGA and our sequencing data were firstly analyzed, respectively. The variant sites of gene expression between cancer and cancerous were screened to obtain the somatic cancerrelated genes by SNV analysis. Based on the functional annotation, the variant sites in which the protein function changed were selected for further study. Next, the shared mutations were aligned into the SNV results of TCGA to calculate the number of samples with the same mutations. We present the following article in accordance with the REMARK reporting checklist (available at https://dx.doi. org/10.21037/tcr-21-1011).

\section{Methods}

\section{Study design}

In order to study the genes and pathways associated with STAD progression, the information from the TCGA database and our own STAD whole genome sequencing (WGS) were comprehensively analyzed. The STAD samples were collected from Shanghai Fengxian District Central Hospital. The study was conducted in accordance with the Declaration of Helsinki (as revised in 2013). The study was approved by the institutional review board of Shanghai Fengxian District Central Hospital (No. 2017KY-13). Informed consent was taken from all the patients. The research design and workflow were shown in Figure 1. The information of STAD-related samples with CNV, RNAseq, and SNV information was downloaded from the TCGA. Then, CNV analysis and differential expression analysis based on the RNAseq data were conducted, respectively, followed by the Venn analysis. Subsequently, the genes in the intersection of the Venn diagram were experienced for survival analysis. In addition, SNV analysis was conducted both on the data from TCGA and our own WGS sequencing data. After Venn analysis, the heatmap was constructed based on the top 20 mutant genes. 


\section{Whole genome resequencing and data preprocessing}

WGS was performed using 2 gastric cancer and 2 adjacent tissues from the patients with STAD based on the BGISEQ-500 sequencer. The raw data was filtered and further mapped to the human reference genome (hg38). Genomic variation, including SNP and InDel, was detected by the HaplotypeCaller of GATK v3.3.0. Besides, strict quality control was applied to the filtered sequencing data, alignment data and mutation results.

\section{Data preprocessing for TCGA}

The copy number information (sample number $\mathrm{n}=442$ ), RNAseq data $(\mathrm{n}=407)$, and somatic mutation (SNV) data $(n=443)$ of STAD, as well as corresponding clinical information, were downloaded from the UCSC Genome Browser Database (https://xenabrowser.net/datapages/). Only STAD samples with CNV, RNAseq, and SNP data were selected for further analysis $(n=363)$. Besides, 32 normal samples with RNASeq data were also studied. The copy number was detected based on the Affymetrix SNP 6.0, mapped to the hy38. The CNV values were calculated followed the formula: $\log 2$ (copy number from Affymetrix SNP 6.0/2). RNAseq values were obtained based on $\log 2$ (fragments per kilobase per million +1 ), and the mRNA was annotated by the gencode.v22 for further analysis (12).

\section{Identification of differentially expressed genes (DEGs) based on the RNAseq data in TCGA}

The DEGs between STAD and normal tissue in the TCGA database were identified through the software of Bayesian method (version 3.38.3, http://www.bioconductor. org/packages/release/bioc/html/limma.html) (13). After analysis, results showed by volcano plot, the threshold of gene expression changed: $\mathrm{P}$ value $<0.05$ and $\log \mathrm{FC}$ (gene expression Fold change) $\geq 1$ (red plot: up-regulated in STAD; green plot: down-regulation in STAD) was constructed.

\section{CNV analysis}

GISTIC 2.0 (version 2.0.23, http://portals.broadinstitute. org/cgi-bin/cancer/publications/pub_paper. cgi?mode=view\&paper_id=216\&P=t) is a data processing module for analyzing cancer CNV (14). Briefly, the copy number segment data of all samples were firstly organized into one file. Then, marker files on the Affymetrix SNP 6.0 were downloaded from TCGA, and CNV was analyzed by GISTIC 2.0, with the amplification/deletion threshold of $0.1, \mathrm{q}$-value $<0.25$, and reference genome $\mathrm{hg} 38$.

\section{The correlation of CNV and DEGs}

By comparing the results of the CNV gene and DEGs, the relationship between gene copy number amplification (amp) and deletion (del), as well as gene up-regulation (up) and down-regulation (down) were showed by the Venn diagram. Besides, the Pearson correlation coefficient between gene copy number and expression levels was calculated. With the threshold of $\mathrm{P}<0.05$, the top 20 genes with the positive correlation between gene expression and CNV were selected as the candidate genes for further analysis.

\section{Functional enrichment analysis based on the candidate genes}

Kyoto Encyclopedia of Genes and Genomes (KEGG) is a knowledge base for systematic analysis of gene functions based on the genes and molecules (15). Gene Ontology (GO), the de facto standard in gene functionality description, is used widely in functional annotation and enrichment analysis (16). GO functions contained three categories: molecular function (MF), cellular component (CC), and biological process (BP). The KEGG and GO enrichment analysis of candidate genes were performed with the threshold of $\mathrm{P}$ value $<0.05$ and gene count $\geq 2$, as the significance. The functional enrichment analysis was carried out by the DAVID (17).

\section{Survival analysis}

The clinical prognosis information including overall survival (OS) was downloaded from TCGA. The survival analysis was used the online software [Survival v2.43-3 package (18)] to combine the prognosis information and candidate genes. The Kaplan-Meier (K-M) curves were carried out with the results of candidate genes related to prognosis. The logrank test and the genes with $\mathrm{P}<0.05$ were considered to be associated with prognosis.

\section{Statistical analysis}

The K-M survival analysis and log-rank test were used to 


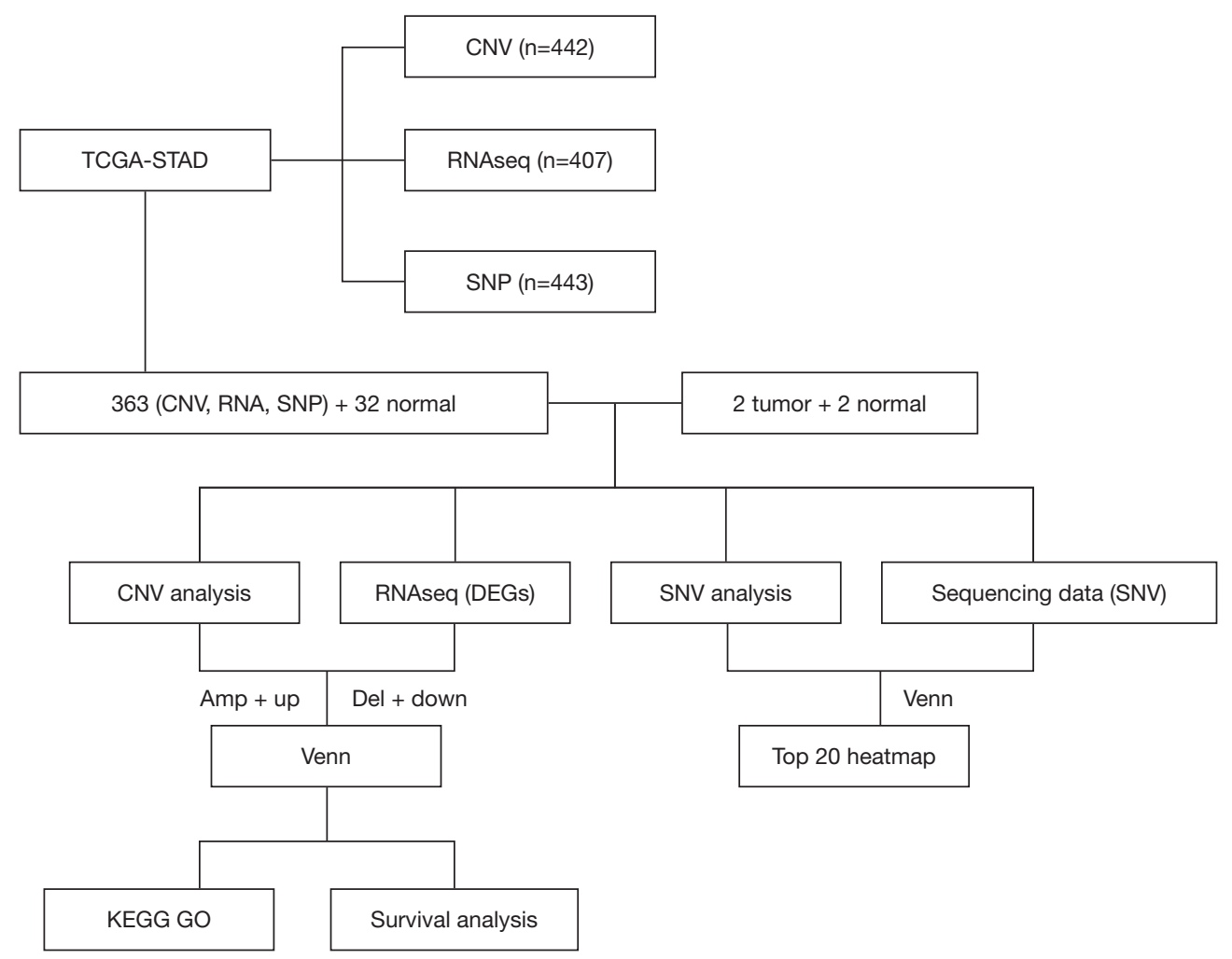

Figure 1 The workflow of this study. STAD, stomach adenocarcinoma; CNVs, copy number variations; SNV, single nucleotide variant; SNP, single nucleotide polymorphism; TCGA, the Cancer Genome Atlas; DEGs, differentially expressed genes.

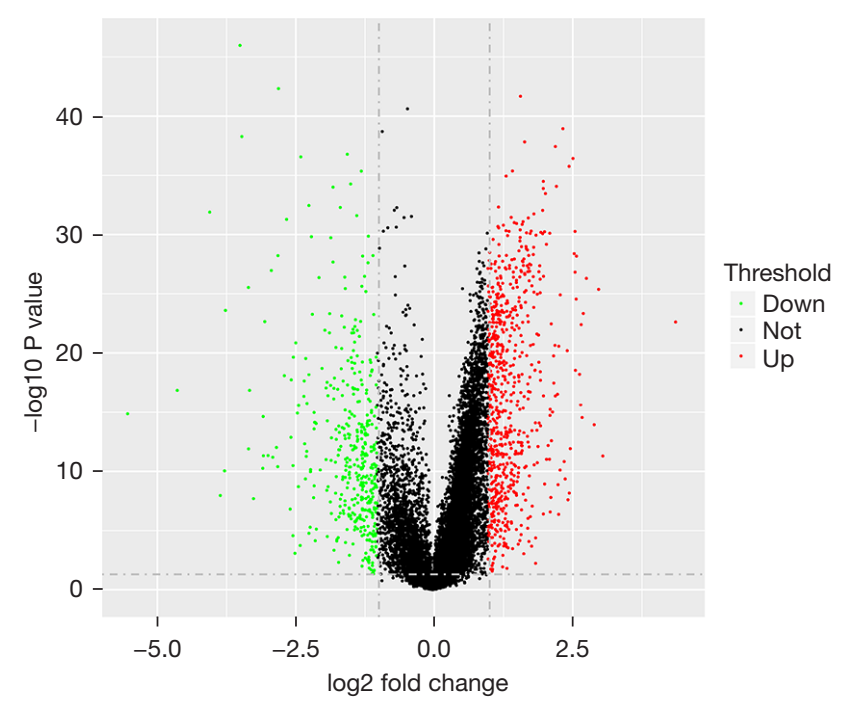

Figure 2 Volcano plot of different expression genes (DEGs) between stomach adenocarcinoma (STAD) and normal in the Cancer Genome Atlas (TCGA) database. Red dots indicate genes that are significantly up-regulated in the tumor group, green dots indicate down-regulated genes, and black dots indicate nonsignificant differential genes. compare the survival trends of categorical variables. $\mathrm{P}<0.05$ would be recognized as significance of other sample, twotailed. All analyses were using one-way analysis of variance (ANOVA; Student's $t$-test) with SigmaPlot 10.0 software (Systat Software Inc., San Jose, CA, USA).

\section{Results}

\section{DEGs between STAD and normal in TCGA}

As shown in Figure 1, we collected gastric cancer and adjacent normal tissues sent to RNAseq. The results showed a threshold of $\mathrm{P}$ value $<0.01$ and $|\log 2(\mathrm{FC})|>1,1,191$ DEGs containing 760 up- and 431 down-regulated genes were identified between gastric cancer and adjacent normal tissues. Figure 2 showed the volcano plot of DEGs based on the expressed values of samples.

\section{CNV analysis based on TCGA}

After we obtained the genes expression changed from RNAseq, we also want to check the genomic DNA copy 

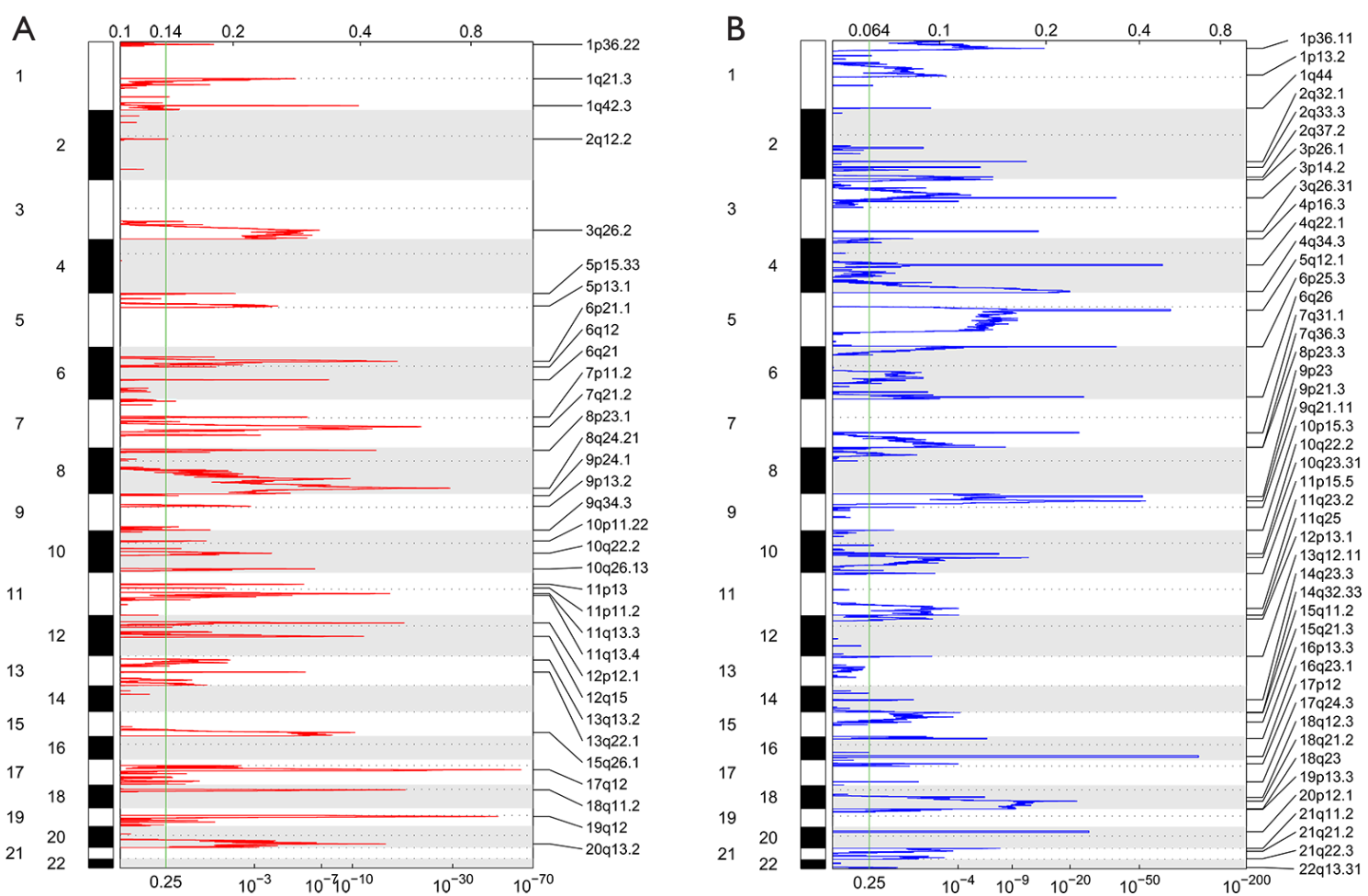

Figure 3 Stomach adenocarcinoma (STAD) tumor copy numbers in genomic DNA compared to a normal baseline. (A) Copy number variations (CNV) amplifications; (B) CNV deletion. The red peak represents amplification and the blue peak represents deletion. The left side is labeled as chromosome, the right side is labeled as a karyotype. The genome is vertically arranged from top to bottom. The top is an amplification or amplification G-scores, and the bottom is q-values.

number by $\mathrm{CNV}$ analysis. Figure 3 showed the genomic DNA copy numbers of STAD tumors compared to a normal baseline. A plot of the G-scores (top) and q-values (bottom) with respect to amplification (Figure $3 \mathrm{~A}$ )/deletion (Figure $3 B$ ) for all markers over the entire region was analyzed. After statistics, we found that the amplifications in $9 p, 17 q$, and $19 q$, and deletions in $4 q, 6 p$, and $18 q$ were significantly altered than others. These findings suggested the genomic DNA copy numbers changed may relate to STAD.

\section{The correlation of CNV and DEGs}

The CNV and DEGs were depicted by the Venn diagram. After we check the results from CNV and DEGs analysis, we found 19 genes with $\mathrm{CNV}$ and alternative expressions with the same trend (11 genes up-regulation and 8 genes down-regulation) compared with DEGs (Figure 4). There were 11 genes with increased copy number and upregulated expression in STAD, 8 genes with decreased copy number and down-regulated expression. Besides, the Pearson correlation coefficient between the copy numbers and expression levels of DEGs was calculated. With the threshold of $\mathrm{P}<0.05$ and based on the ranking of Pearson, the top 20 genes were selected as the candidate gene set for further study (Table 1).

\section{Functional enrichment analysis}

Following our results obtained that genes expression changed, we also want to know these genes involved in which MFs. Table 2 showed the KEGG and GO enrichment analysis results based on the above top 20 genes. We found that these genes were mainly enriched in the human-related (hsa) DNA replication pathway (ID: hsa 03030) and vitamin digestion and absorption pathways (ID: hsa 04977). Moreover, we also found these genes involved in several biological functions according to the GO analysis, such as cell adhesion, epidermis development, digestive tract development, and serine-type 


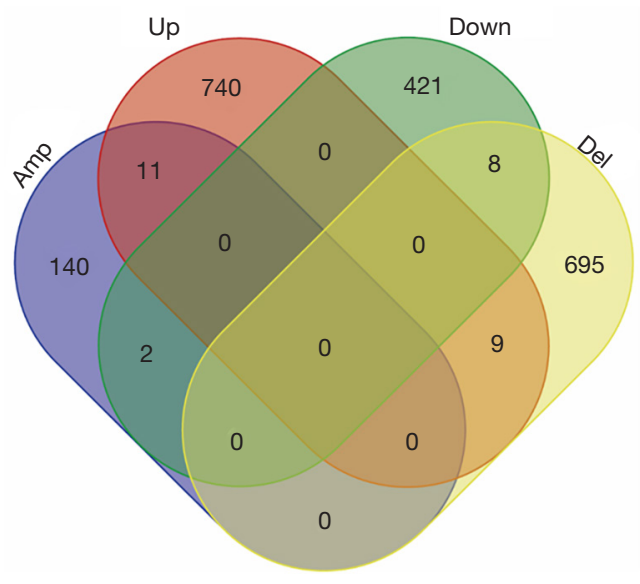

Figure 4 Venn diagram based on the comparison of copy number variations $(\mathrm{CNV})$ and different expression genes (DEGs). The genes with increased copy number and up-regulated expression (11) were CAMP Responsive Element Binding Protein 3 Like 1 (CREB3L1), replication factor C subunit 3 (RFC3), cyclin E1 (CCNE1), chaperonin containing TCP1 subunit 2 (CCT2), cell division protein kinase 6 (CDK6), cyclin D1 (CCND1), thyroid hormone receptor interactor 13 (TRIP13), SLC12A7, Krüppellike zinc-finger transcription factor 5 (KLF5), lysozyme (LYZ), and torsin family 4 member A (TOR4A). The genes with decreased copy number and down-regulated expression (8) were crystallin alpha B (CRYAB), neural cell adhesion molecule 1 (NCAM1), transmembrane protein 252 (TMEM252), zinc finger and BTB domain containing 16 (ZBTB16), S100 calcium binding protein B (S100B), MAF, phosphoglucomutase 5 (PGM5), and calsequestrin 2 (CASQ2). The samples for the up-regulated group (up, 740 genes) and Down-regulated group (down, 421 genes) were from TCGA; the samples for Amp group (140 genes) and Del group (695 genes) were collected by authors.

peptidase activity.

\section{Survival analysis}

After we identify the candidate genes from gastric cancer tissue, we also check the survival rate by prognostic survival analysis which was performed on 327 samples based on the expression level of candidate genes. Previous research indicated that proline-relate genes correlated with cell survival via PI3K/Akt signaling pathway (19). Our results obtained that the threshold of $\mathrm{P}<0.05$, proline rich 15 like ( $\mathrm{PRR} 15 \mathrm{~L}, \mathrm{P}=0.0047)$, human riboflavin transporter-3 (SLC52A3, P=0.00071) and spindle and kinetochore
Table 1 The top 20 mutant genes based on the correlation of CNV and DEGs

\begin{tabular}{|c|c|c|c|}
\hline CNV & DEGs & $r$ & $P$ value \\
\hline CCKBR & $C C K B R$ & 0.191126 & 0.000250 \\
\hline DNA2 & DNA2 & 0.15071 & 0.004002 \\
\hline MCM6 & MCM6 & 0.148543 & 0.004566 \\
\hline GPR35 & GPR35 & 0.144973 & 0.005654 \\
\hline GPR155 & GPR155 & 0.142873 & 0.006397 \\
\hline PRSS22 & PRSS22 & 0.140763 & 0.007231 \\
\hline FAT1 & FAT1 & 0.135892 & 0.009536 \\
\hline PRR15L & PRR15L & 0.132366 & 0.011592 \\
\hline $\mathrm{P} 3 \mathrm{H} 2$ & $\mathrm{P} 3 \mathrm{H} 2$ & 0.130432 & 0.012878 \\
\hline GPATCH4 & GPATCH4 & 0.130406 & 0.012896 \\
\hline SPRR1A & SPRR1A & 0.128775 & 0.014078 \\
\hline APOBEC2 & APOBEC2 & 0.126177 & 0.016158 \\
\hline COLGALT1 & COLGALT1 & 0.123115 & 0.018951 \\
\hline ITGA6 & ITGA6 & 0.121876 & 0.020195 \\
\hline FAP & $F A P$ & 0.120831 & 0.021299 \\
\hline SLC52A3 & SLC52A3 & 0.120572 & 0.021581 \\
\hline FAM84B & $F A M 84 B$ & 0.120005 & 0.022208 \\
\hline RNASEH $2 A$ & RNASEH $2 A$ & 0.118851 & 0.023535 \\
\hline$S K A 1$ & SKA1 & 0.117382 & 0.025322 \\
\hline$R B P 2$ & $R B P 2$ & 0.116428 & 0.026545 \\
\hline
\end{tabular}

CNV, copy number variation; DEG, different expression gene.

associated complex subunit 1 (SKA1, $\mathrm{P}=0.035$ ). Moreover, we also check the survival rate whether related to 20 DEGs expressions and discovered that only PRR15L, SLC52A3 and SKA1 showed a significant difference between high expression and low expression (Table S1). Taken together, PRR15L and SLC52A3 were considered to be closely related to the prognosis of STAD. Figure 5 showed that the high levels of PRR15L (Figure 5A), SLC52A3 (Figure 5B) and SKA1 (Figure 5C) in STAD patients were positively correlated with the better survival rate.

\section{SNV analysis}

Based on our WGS data, the SNV of these paired samples from STAD tissue and normal tissue were screened out and matched to the SNV in TCGA. Figure 6 showed the 
Table 2 Enrichment analysis based on the candidate genes

\begin{tabular}{lll}
\hline Category & Term & Genes \\
\hline GO_BP & GO:0043137-DNA replication, removal of RNA primer & DNA2, RNASEH2A \\
& GO:0006260-DNA replication & DNA2, RNASEH2A, MCM6 \\
& GO:0048565-digestive tract development & ITGA6, CCKBR \\
& GO:0007155-cell adhesion & ITGA6, FAT1, FAP \\
& GO:0008544-epidermis development & SPRR1A, RBP2 \\
& GO:0016337-single organismal cell-cell adhesion & ITGA6, FAT1 \\
GO_CC & GO:0005925-focal adhesion & ITGA6, FAT1, FAP \\
& GO:0030175-filopodium & ITGA6, FAT1 \\
& GO:0005604-basement membrane & P3H2, ITGA6 \\
GO_MF & GO:0003678-DNA helicase activity & DNA2, MCM6 \\
& GO:0008236-serine-type peptidase activity & FAP, PRSS22 \\
KEGG & hsa03030: DNA replication & DNA2, RNASEH2A, MCM6
\end{tabular}

BP, biological process; CC, cellular component; GO, Gene Ontology; KEGG, Kyoto Encyclopedia of Genes and Genomes; MF, molecular function.

A
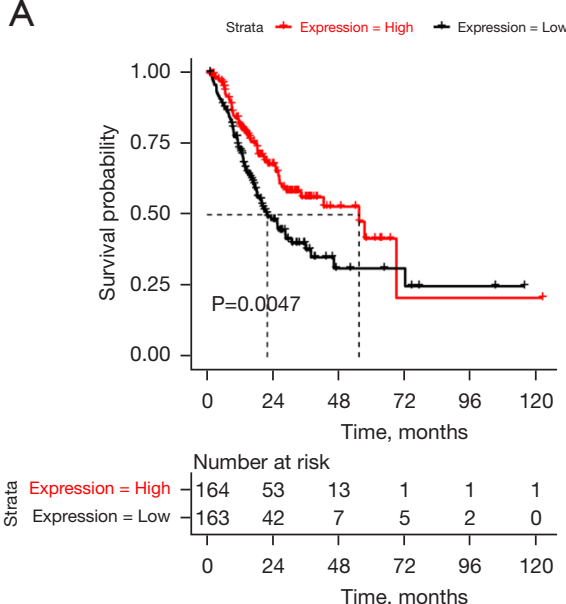

B
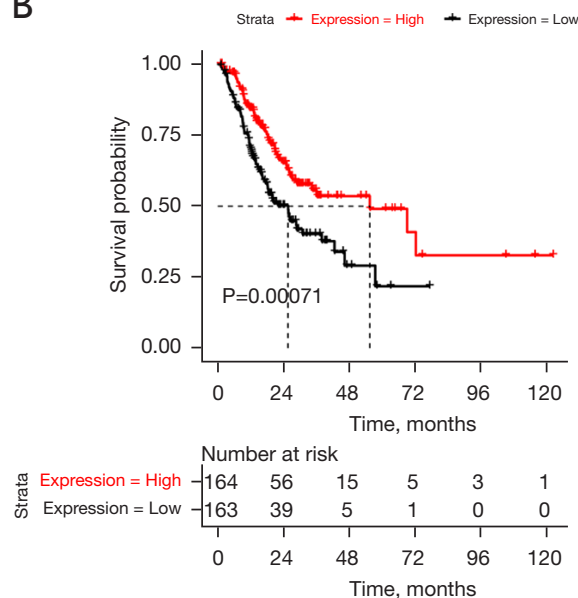

C
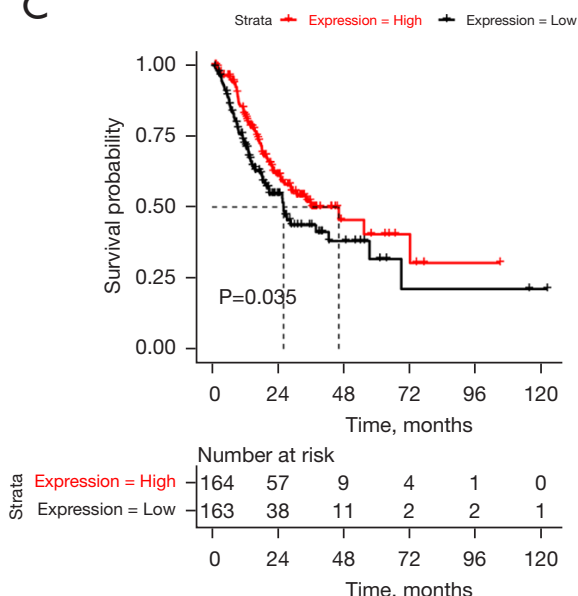

Figure 5 The Kaplan-Meier survival curves of PRR15L, SLC52A3 and SKA1 on the stomach adenocarcinoma (STAD). The high levels of PRR15L ( $\mathrm{P}=0.0047)(\mathrm{A})$, SLC52A3 ( $\mathrm{P}=0.00071)(\mathrm{B})$ and SKA1 $(\mathrm{P}=0.035)(\mathrm{C})$ were positively related to the better survival rate of STAD.

heatmap for the top 20 mutant genes based on the number of mutant samples. The top 3 genes were the Titin gene (TTN, 237 samples), Mucin (MUC16, 152 samples), and Filaggrin (FLG, 108 samples). The results indicated that TTN, MUC16, and FLG might closely relate to the STAD progression. Moreover, the PCDHB 8 and HYDIN genes from the top 20 mutant genes showed a negative correlation with OS in patients. The patients with low expression of PCDHB8 and HYDIN genes showed a better OS rate than patients with high expression of PCDHB 8 and HYDIN (Figure $6 B, 6 C$ ). In addition, we also analyzed the functional enrichment for the top 20 mutant genes by KEGG analysis (Table S2). These findings suggested that the mutant genes highly correlated with STAD progression. 


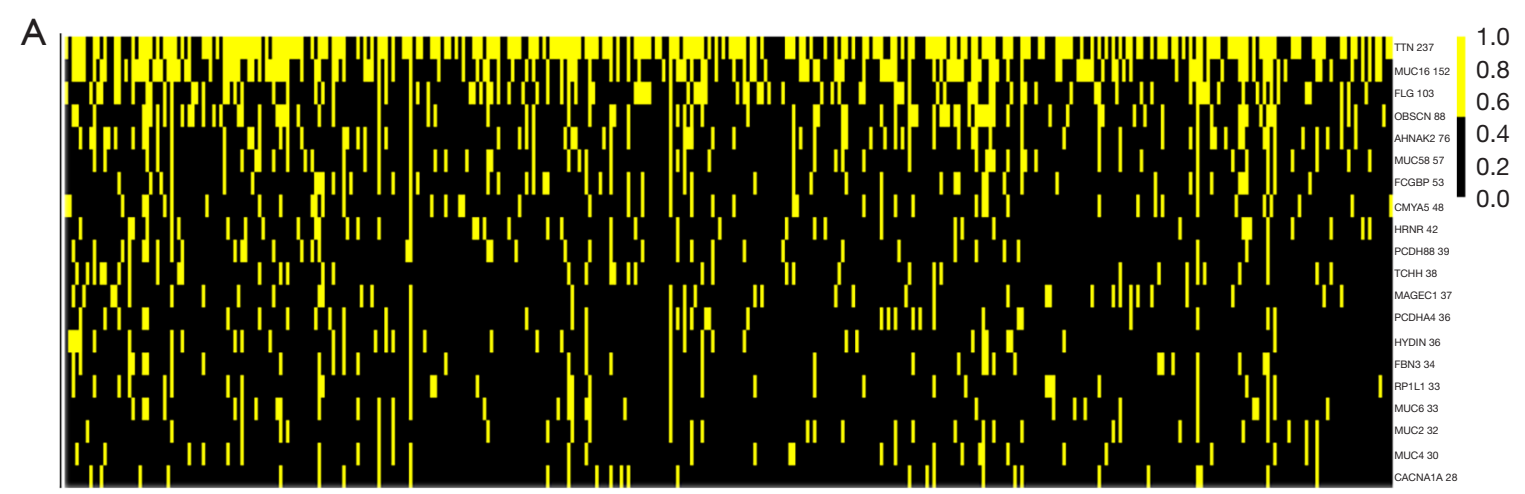

B

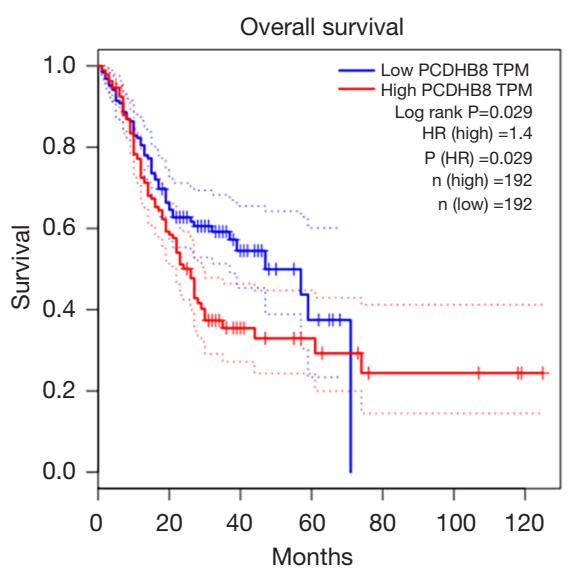

C

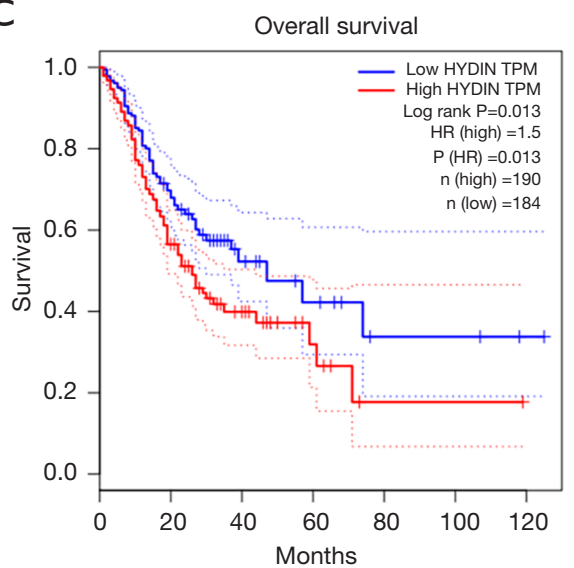

Figure 6 Heat map for mutation genes from the Cancer Genome Atlas (TCGA) database. (A) The mutation sites spectrum of top 20 samples based on the TCGA stomach adenocarcinoma (STAD) data and our own sequencing data. The ordinate represents the gene in which the mutation occurred, and the abscissa represents the samples in the TCGA. Yellow represents genetic variation. (B) Low expression of PCDHB8 increased the overall survival rate in STAD patients. (C) Low expression of HYDIN enhanced the overall survival rate in STAD patients. Numbers of sample from TCGA: 363 tumor samples and 32 normal samples. HR, hazard ratio; TPM, transcripts per million.

\section{Discussion}

There were 1,191 DEGs containing 760 up- and 431 down-regulated genes between gastric cancer and adjacent tissues based on the TCGA STAD data. Combined with the TCGA database and our DEGs results mainly enriched in the DNA replication, cell adhesion, digestive tract development, and serinetype peptidase activity pathway or related functions. Moreover, survival analysis showed that PRR15L and SLC52A3 were positively related to the long life span. In addition, according to the SNV analysis, TTN, MUC16, and FLG showed similar mutations in most samples in TCGA STAD data and our own samples, indicating that the TTN, MUC16, and FLG were probably involved in the development of gastric cancer.
Cell-adhesion related molecules not only play adhesive functions but also regulate signal-transduction pathways by interacting with molecules (20). Plenty of experimental evidence has indicated that cell-adhesion processes play an important role in tumorigenesis, in particular in invasion and metastasis (21). Alexiou et al. evaluated serum concentrations of soluble cell adhesion molecules in STAD patients and control. The results showed that the serum levels of E-selectin, intercellular adhesion molecule-1, and vascular cell adhesion molecule-1 in gastric cancer patients were significant prognostic factors for patient survival (22). Moreover, based on the sequencing of the exomes of gastric adenocarcinomas and matched normal DNAs, the mutated genes were mainly involved in the cell adhesion biological pathway among the frequently (23), which was consistent with our results. 
SLC52A3 gene coded the human riboflavin transporter-3 and disruption of Slc52a3 gene caused newborn mice death due to riboflavin deficiency (24). Numerous studies have shown that SNP of SLC52A3 might be a key biomarker of cancer patients. For example, the single nucleotide rs13042395 polymorphism in SLC52A3 gene is associated with regional lymph node metastasis and relapse-free survival in esophageal squamous cell carcinoma patients (25). It is well known that gene-environment interactions may increase STAD risk. Based on the susceptibility loci identified by genome-wide association studies suggest that SLC52A3 rs13042395 showed an interaction with alcohol drinking in risk of STAD in the Chinese population (26). In our study, SLC52A3 was a key prognostic factor for STAD and a high level of SLC52A3 was positively related to the long life span. In addition, PRR15 was another prognostic factor for STAD. Gates et al. has shown increased PRR15 transcription in colorectal cancers with mutations in the adenomatous polyposis coli (Apc) protein (27). However, there were few studies on the relationship between SKA1 and STAD. So, it is reasonable to speculate that the occurrence, development and prognosis of STAD may be closely related to SKA1, which requires further experimental verification.

The CNV analysis indicated that the amplifications in $9 \mathrm{p}, 17 \mathrm{q}$, and $19 \mathrm{q}$, and deletions in $4 \mathrm{q}, 6 \mathrm{p}$, and $18 \mathrm{q}$ were more significant. More interestingly, the most frequent amplifications and deletions were consistent with previously published studies (28). In addition, the SNV analysis based on the TCGA STAD data and our own sequencing data indicated that the TTN, MUC16, and FLG were probably involved in the development of gastric cancer. Mucin 16 (cancer antigen 125) is a cell surface glycoprotein that plays a role in promoting cancer cell growth in ovarian cancer (29). Besides, the FLG rs2065955 polymorphism was significantly related to the susceptibility to Epstein-Barr virus-associated gastric carcinoma (30). Our results were consistent with previous studies.

In conclusion, we have shown that cell adhesion appears to be a critical component in the BPs of STAD. Cell adhesion was involved in the BP of STAD. Besides, PRR15L and SLC52A3 were the potential prognostic biomarkers for STAD, and high levels of PRR15L and SLC52A3 were positively related to the long life span. In addition, TTN, MUC16, and FLG were involved in the development of STAD, these genes may serve as potential targets for gene therapy in STAD.

\section{Acknowledgments}

Funding: This paper is supported by the department: Shanghai Municipal Health Commission (201740141)

\section{Footnote}

Reporting Checklist: The authors have completed the REMARK reporting checklist. Available at https://dx.doi. org/10.21037/tcr-21-1011

Data Sharing Statement: Available at https://dx.doi. org/10.21037/tcr-21-1011

Conflicts of Interest: All authors have completed the ICMJE uniform disclosure form (available at https://dx.doi. org/10.21037/tcr-21-1011). The authors have no conflicts of interest to declare.

Ethical Statement: The authors are accountable for all aspects of the work in ensuring that questions related to the accuracy or integrity of any part of the work are appropriately investigated and resolved. The study was conducted in accordance with the Declaration of Helsinki (as revised in 2013). The study was approved by the institutional review board of Shanghai Fengxian District Central Hospital (No. 2017-KY-13). Informed consent was taken from all the patients.

Open Access Statement: This is an Open Access article distributed in accordance with the Creative Commons Attribution-NonCommercial-NoDerivs 4.0 International License (CC BY-NC-ND 4.0), which permits the noncommercial replication and distribution of the article with the strict proviso that no changes or edits are made and the original work is properly cited (including links to both the formal publication through the relevant DOI and the license). See: https://creativecommons.org/licenses/by-nc-nd/4.0/.

\section{References}

1. Wu M, Xia Y, Wang Y, et al. Development and validation of an immune-related gene prognostic model for stomach adenocarcinoma. Biosci Rep 2020;40:BSR20201012.

2. Qin G, Yang L, Ma Y, et al. The exploration of diseasespecific gene regulatory networks in esophageal carcinoma and stomach adenocarcinoma. BMC Bioinformatics 2019;20:717. 
3. Huo Q. Analysis of expression profile of miRNA in stomach adenocarcinoma. J BUON 2017;22:1154-9.

4. Neapolitan R, Horvath CM, Jiang X. Pan-cancer analysis of TCGA data reveals notable signaling pathways. BMC Cancer 2015;15:516.

5. Andor N, Graham TA, Jansen M, et al. Pan-cancer analysis of the extent and consequences of intratumor heterogeneity. Nat Med 2016;22:105-13.

6. Weisenberger DJ. Characterizing DNA methylation alterations from The Cancer Genome Atlas. J Clin Invest 2014;124:17-23.

7. Hackmann K, Kuhlee F, Betcheva-Krajcir E, et al. Ready to clone: CNV detection and breakpoint fine-mapping in breast and ovarian cancer susceptibility genes by high-resolution array CGH. Breast Cancer Res Treat 2016;159:585-90.

8. Ewing AD, Gacita A, Wood LD, et al. Widespread somatic L1 retrotransposition occurs early during gastrointestinal cancer evolution. Genome Res 2015;25:1536-45.

9. Kambayashi Y, Fujimura T, Ohuchi K, et al. Advanced Invasive Extramammary Paget's Disease Concomitant with Cecal Cancer Possessing Rare Variant of TP53 Single Nucleotide Polymorphism. Case Rep Oncol 2019;12:855-60.

10. Cai H, Jing C, Chang X, et al. Mutational landscape of gastric cancer and clinical application of genomic profiling based on target next-generation sequencing. J Transl Med 2019; 17:189.

11. Min P, Li W, Zeng D, et al. A single nucleotide variant in microRNA-1269a promotes the occurrence and process of hepatocellular carcinoma by targeting to oncogenes SPATS2L and LRP6. Bull Cancer 2017;104:311-20.

12. Harrow J, Frankish A, Gonzalez JM, et al. GENCODE: the reference human genome annotation for The ENCODE Project. Genome Res 2012;22:1760-74.

13. Smyth GK. limma: Linear Models for Microarray Data. In: Gentleman R, Carey VJ, Huber W, et al. editors. Bioinformatics and Computational Biology Solutions Using $\mathrm{R}$ and Bioconductor. Statistics for Biology and Health. New York, NY: Springer, 2005.

14. Mermel CH, Schumacher SE, Hill B, et al. GISTIC2.0 facilitates sensitive and confident localization of the targets of focal somatic copy-number alteration in human cancers. Genome Biol 2011;12:R41.

15. Kanehisa M, Goto S. KEGG: kyoto encyclopedia of genes and genomes. Nucleic Acids Res 2000;28:27-30.

16. Du Z, Zhou X, Ling Y, et al. agriGO: a GO analysis toolkit for the agricultural community. Nucleic Acids Res
2010;38:W64-70.

17. Dennis G Jr, Sherman BT, Hosack DA, et al. DAVID: Database for Annotation, Visualization, and Integrated Discovery. Genome Biol 2003;4:P3.

18. Therneau T, Lumley T. Survival: Survival analysis, including penalised likelihood. R package version 2.35-7. Vienna, Austria: R Foundation for Statistical Computing, 2009.

19. Wang H, Zhang Q, Wen Q, et al. Proline-rich Akt substrate of 40kDa (PRAS40): a novel downstream target of PI3k/Akt signaling pathway. Cell Signal 2012;24:17-24.

20. Saitoh M. Involvement of partial EMT in cancer progression. J Biochem 2018;164:257-64.

21. Cavallaro U, Christofori G. Cell adhesion and signalling by cadherins and Ig-CAMs in cancer. Nat Rev Cancer 2004;4:118-32.

22. Alexiou D, Karayiannakis AJ, Syrigos KN, et al. Clinical significance of serum levels of E-selectin, intercellular adhesion molecule-1, and vascular cell adhesion molecule-1 in gastric cancer patients. Am J Gastroenterol 2003;98:478-85.

23. Zang ZJ, Cutcutache I, Poon SL, et al. Exome sequencing of gastric adenocarcinoma identifies recurrent somatic mutations in cell adhesion and chromatin remodeling genes. Nat Genet 2012;44:570-4.

24. Yoshimatsu H, Yonezawa A, Yamanishi K, et al. Disruption of Slc52a3 gene causes neonatal lethality with riboflavin deficiency in mice. Sci Rep 2016;6:27557.

25. Tan HZ, Wu ZY, Wu JY, et al. Single nucleotide polymorphism rs13042395 in the SLC52A3 gene as a biomarker for regional lymph node metastasis and relapsefree survival of esophageal squamous cell carcinoma patients. BMC Cancer 2016;16:560.

26. Cai M, Dai S, Chen W, et al. Environmental factors, seven GWAS-identified susceptibility loci, and risk of gastric cancer and its precursors in a Chinese population. Cancer Med 2017;6:708-20.

27. Gates KC. The role of proline rich 15 in trophoblast cell development. Dissertations and TheseGradworks, 2012.

28. Wang K, Yuen ST, Xu J, et al. Whole-genome sequencing and comprehensive molecular profiling identify new driver mutations in gastric cancer. Nat Genet 2014;46:573-82.

29. Streppel MM, Vincent A, Mukherjee R, et al. Mucin 16 (cancer antigen 125) expression in human tissues and cell lines and correlation with clinical outcome in 
adenocarcinomas of the pancreas, esophagus, stomach, and colon. Hum Pathol 2012;43:1755-63.

30. Kuang X, Sun L, Liu S, et al. Association of single nucleotide polymorphism rs2065955 of the filaggrin gene

Cite this article as: Wei GG, Geng XC, Fu MS, Wei J, Yu HB, Li XH, Gao GL. Molecular signature of gastric cancer progression in clinical using whole genome sequencing and the Cancer Genome Atlas (TCGA) analysis. Transl Cancer Res 2021. doi: $10.21037 /$ tcr-21-1011 with susceptibility to Epstein-Barr virus-associated gastric carcinoma and EBV-negative gastric carcinoma. Virol Sin 2016;31:306-13. 
Supplementary

Table S1 Survival rate of the 20 different expression genes

\begin{tabular}{lcccc}
\hline No. & Gene & P value & High.median & Low.median \\
\hline 1 & APOBEC2 & 0.414248 & 27.33 & 35.97 \\
2 & CCKBR & 0.348236 & 26.08 & 35.97 \\
3 & COLGALT1 & 0.639339 & 30.88 & 28.55 \\
4 & DNA2 & 0.084844 & 42.51 & 26.02 \\
5 & FAM84B & 0.088592 & 55.39 & 26.08 \\
6 & FAP & 0.147319 & 26.31 & 46.22 \\
7 & FAT1 & 0.054411 & 42.51 & 22.17 \\
8 & GPATCH4 & 0.82674 & 28.94 & 28.55 \\
9 & GPR155 & 0.761801 & 28.55 & 30.88 \\
10 & GPR35 & 0.059915 & 46.22 & 26.08 \\
11 & ITGA6 & 0.05153 & 46.22 & 25.69 \\
12 & MCM6 & 0.935766 & 34.26 & 27.33 \\
13 & P3H2 & 0.154286 & 26.08 & 42.51 \\
14 & PRR15L & 0.004659 & 55.39 & 21.98 \\
15 & PRSS22 & 0.268143 & 35.97 & 28.55 \\
16 & RBP2 & 0.699066 & 30.88 & 28.94 \\
17 & RNASEH2A & 0.194839 & 35.97 & 26.31 \\
18 & SKA1 & 0.034646 & 46.22 & 26.08 \\
19 & SLC52A3 & 0.000707 & 55.39 & 25.59 \\
20 & SPRR1A & 0.478222 & 34.26 & 28.55 \\
\hline & & &
\end{tabular}


Table S2 KEGG analysis for top 20 mutant genes

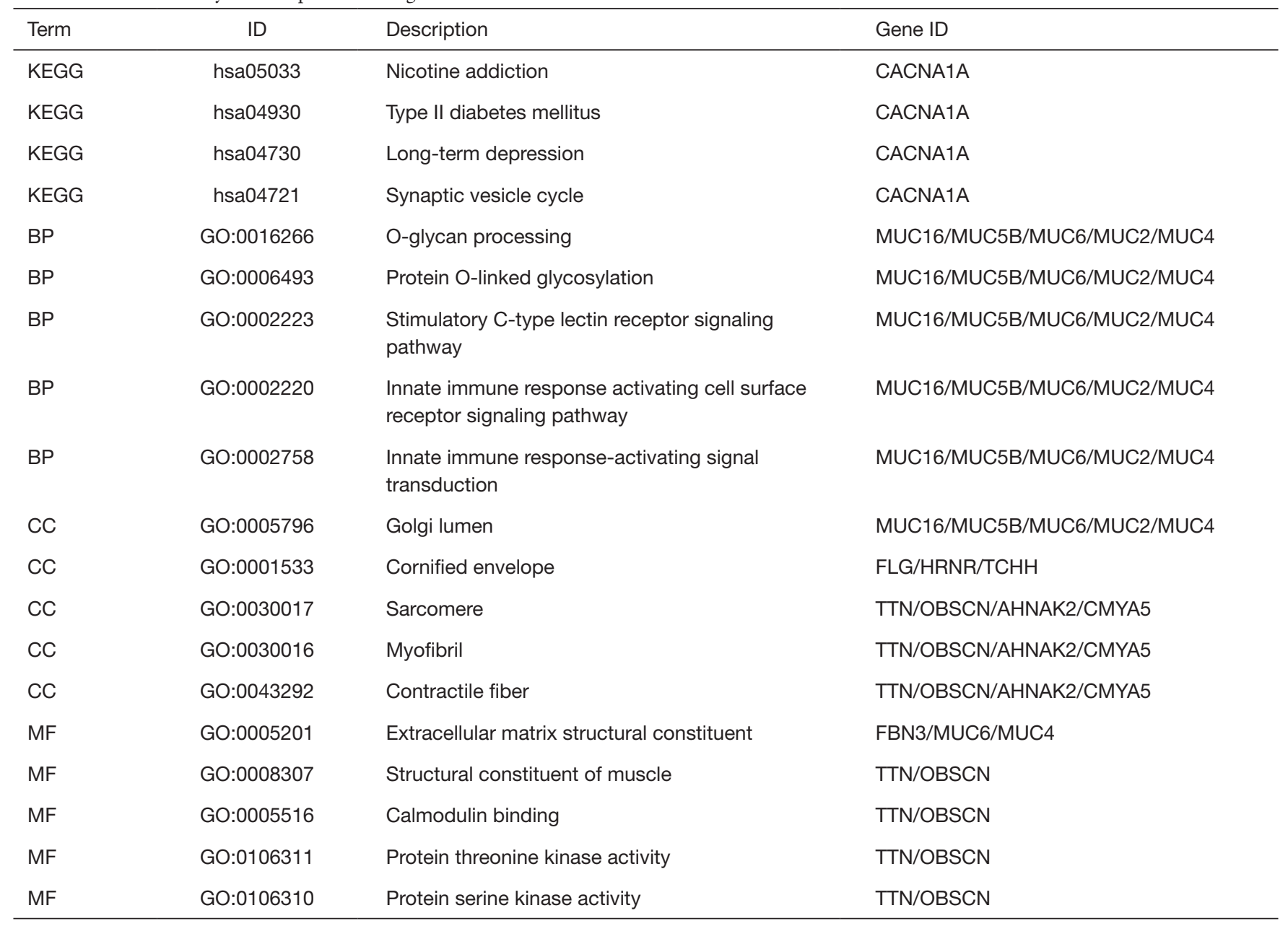

BP, biological process; CC, cellular component; GO, Gene Ontology; KEGG, Kyoto Encyclopedia of Genes and Genomes; MF, molecular function. 